\title{
6 Weitergabe von Informationen an „mitwirkende Personen“ im Rahmen der medizinischen Forschung
}

Das folgende Kapitel beantwortet die Forschungsfrage 4.6:

Nach dem Entwurfeines Gesetzes zur Neuregelung des Schutzes von Geheimnissen bei der Mitwirkung Dritter an der Berufsausübung schweigepflichtiger Personen (BR-Drucksache 163/17) soll \$203 StGB in der Form geändert werden, dass die Weitergabe von Informationen an „mitwirkende Personen “für einen Berufsgeheimnisträger (z.B. Arzt) straflos sein soll, wenn diese an der ,beruflichen Tätigkeit“ des Berufsgeheimnisträgers mitwirken. Prüfen Sie bitte, ob eine Mitwirkung an einer Forschungstätigkeit eines Arztes auch als Mitwirkungshandlung im Sinne der neuen Rechtsvorschrift verstanden werden kann. Gehen Sie bitte daraufein, wie die „berufliche Tätigkeit" eines Arztes auch mit Blick aufdie EU-DSGVO und den bisherigen Rechtsrahmen definiert wird und ob Unterschiede zwischen der ärztlichen Tätigkeit in einem Universitätskrankenhaus/sonstigem Krankenhaus und einer Arztpraxis besteht.

Das Gesetz zur Neuregelung des Schutzes von Geheimnissen bei der Mitwirkung Dritter an der Berufsausübung schweigepflichtiger Personen bewirkt in seinem Art. 1 eine wichtige Änderung des $\$ 203$ StCB. ${ }^{418}$ 


\subsection{Die Neuregelung des $\$ 203$ StGB}

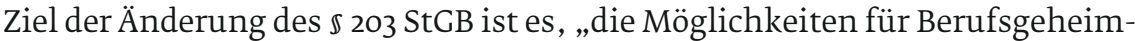
nisträger zu erweitern, sich im Rahmen ihrer beruflichen oder dienstlichen Tätigkeit ohne (straf-)rechtliches Risiko der Mitwirkung dritter Personen zu bedienen“. ${ }^{419}$

Strafbar macht sich nach $\mathbb{} 203$ StGB, wer unbefugt ein fremdes Geheimnis offenbart, das ihm als Berufsgeheimnisträger im Sinn von Abs. 1 und 2 anvertraut worden oder sonst bekannt geworden ist. Für diese bestand in der Hinzuziehung von Personen, die nicht ebenfalls der Strafbarkeit von $\mathbb{} 203$ StGB unterfallen, ein rechtliches Risiko. ${ }^{420}$ Die herrschende Meinung ging davon aus, dass nicht einem eng auszulegenden Gehilfenbegriff unterfallende Personen nicht zur Sphäre des Berufsgeheimnisträgers zählen ${ }^{421}$ und damit nicht am Vertrauensverhältnis teilnehmen. ${ }^{422}$ Andere argumentierten, der Begriff des berufsmäßig tätigen Gehilfen könne weit ausgelegt werden und umfasse etwa auch externe IT-Dienstleister. ${ }^{223}$ Die Hinzuziehung insbesondere eines externen Dienstleisters barg mithin das Risiko einer Strafbarkeit nach $\mathbb{2 0 3}$ StGB. ${ }^{224}$ Zudem bestand kein strafrechtlicher Schutz für die externen Personen anvertrauten oder bekannt gewordenen Geheimnisse.

Die zunehmende Nutzung von Informationstechnik macht es häufig aber erforderlich, externe Fachkräfte hinzuzuziehen, die außerhalb der Sphäre des Berufsgeheimnisträgers stehen, beispielsweise zur Wartung der eingesetzten Technik. Dabei können diesen Personen jedoch fremde Geheimnisse offenbart werden. Zudem wird zunehmend die eigene Informationstechnik durch externe Technik ergänzt, beispielsweise in Form des Cloud Computing. Der Weg über eine informierte Einwilligung des Berechtigten führte zwar zu einem Ausschluss der Strafbarkeit nach $\$ 203$ StGB, ${ }^{425}$ da in diesem Fall eine Befugnis vorliegt. ${ }^{426}$ Deren Einholung ist aber nicht immer möglich und mit Aufwand

419 BT-Drs. 18/12940, 1.

420 S. z.B. Behling u.a. 2015, 9ff., 15; Jandt/Roßnagel, MedR 2011, $140 \mathrm{ff}$.

421 Die Gesetzesbegründung sieht in der Einbindung externer Personen eine Überdehnung des Begriffs „Gehilfe“;

BT-Drs. 18/11936, 18. Der Begriff sei der Gedanke eines „geschlossenen Geheimnisträgerkreises immanent“. „EXterne Personen, die selbständig tätig sind oder die in den Betrieb eines Dritten eingebunden sind, sind deshalb regelmäßig keine Gehilfen".

422 So auch die Gesetzesbegründung, BT-Drs. 18/11936, 18; Lenckner/Eisele, in: Schönke/Schröder, $\$ 203$ Rn. 19b; Kargl, in: Kindhäuser/Neumann/Paeffgen, \ 203 Rn. 21; Cierniak/Niehaus, in: MüKo StGB, \ 203 Rn. 54, 56; bezogen auf Cloud-Anbieter Wicker 2016, 136ff.

423 S. auch Kargl, in: Kindhäuser/Neumann/Paeffgen, § 203 Rn. 38a, Gehilfe, sofern beim Outsourcing „die organisatorische Anbindung der Datenverwalter an den Auftraggeber gewährleistet“ ist. Nach Cierniak/Niehaus, in: MüKo StGB, § 203 Rn. 129, Gehilfe, wenn die Aufträge „eine besonders enge Beziehung zum Aufgabenbereich des Schweigepflichtigen aufweisen".

424 S. etwa LG Flensburg, Urteil vom 5.7.2013 - 40 54/11; s. zum Outsourcing im Gesundheitsbereich Jandt/Roßnagel/Wilke, NSR 2011, 641ff.

425 S. z.B. Jandt/Roßnagel/Wilke, RDV 2011, 222 (228); Jandt/Roßnagel/Wilke, NSR 2011, 641.

426 Man beachte aber den Streit, ob die Einwilligung das Tatbestandsmerkmal „unbefugt“ ausschließt oder die Rechtswidrigkeit entfallen lässt, s. hierzu Cierniak/Niehaus, in: MüKo StGB, § 203 Rn. 58ff. 
6 Weitergabe von Informationen an „mitwirkende Personen“ im Rahmen der medizinischen Forschung

verbunden. ${ }^{27}$ Zudem würde dieser Weg eine nach vorliegender Einwilligung und verweigerter Einwilligung differenzierte Datenverarbeitung erfordern. Auch die Annahme einer konkludenten Einwilligung 428 barg rechtliche Risiken; die Weitergabe von Geheimnissen zu Forschungszwecken kann sie zudem nicht legitimieren. ${ }^{429}$ Zahlreiche Meinungsstreitigkeiten in der strafrechtlichen Fachliteratur zu $\mathbb{2} 203$ StGB und fehlende obergerichtliche Rechtsprechung zum Thema Outsourcing verkomplizierten die Sache weiter und sorgten für Rechtsunsicherheit. ${ }^{430}$ In der Praxis wurde teilweise versucht, die Problematik durch den Abschluss von Mehrfach-Arbeitsverhältnissen zu lösen. ${ }^{431}$

Die Novelle des $\$ 203$ StGB soll hier nun Rechtsklarheit für alle Beteiligten herstellen und den Schutz von Privatgeheimnissen auch in einer digitalisierten Arbeitswelt garantieren.

\subsubsection{Der neue $§ 203$ StGB}

In $₫ 203$ StGB wurde der bisherige Abs. 2a aufgehoben und durch die neuen Abs. 3 und 4 ersetzt. Diese lauten:

„(3) Kein Offenbaren im Sinne dieser Vorschrift liegt vor, wenn die in den Absätzen 1 und 2 genannten Personen Geheimnisse den bei ihnen berufsmäßig tätigen Gehilfen oder den bei ihnen zur Vorbereitung auf den Beruftätigen Personen zugänglich machen. Die in den Absätzen 1 und 2 Genannten dürfen fremde Geheimnisse gegenüber sonstigen Personen offenbaren, die an ihrer beruflichen oder dienstlichen Tätigkeit mitwirken, soweit dies für die Inanspruchnahme der Tätigkeit der sonstigen mitwirkenden Personen erforderlich ist; das Gleiche gilt für sonstige mitwirkende Personen, wenn diese sich weiterer Personen bedienen, die an der beruflichen oder dienstlichen Tätigkeit der in den Absätzen 1 und 2 Genannten mitwirken.

(4) Mit Freiheitsstrafe bis zu einem Jahr oder mit Geldstrafe wird bestraft, wer unbefugt ein fremdes Geheimnis offenbart, das ihm bei der Ausübung oder bei Gelegenheit seiner Tätigkeit als mitwirkende Person oder als bei den in den Absätzen 1 und 2 genannten Personen tätiger Beauftragter für den Datenschutz bekannt geworden ist. Ebenso wird bestraft, wer

1. als in den Absätzen 1 und 2 genannte Person nicht dafür Sorge getragen hat, dass eine sonstige mitwirkende Person, die unbefugt ein fremdes, ihr bei der Ausübung oder bei Gelegenheit ihrer Tätigkeit bekannt gewordenes Geheimnis offenbart, zur Geheimhaltung

427 Die Gesetzesbegründung nennt etwa die Archivierung von Altbeständen von Daten; BT-Drs. 18/11936, 18. Zu den Problemen der Einwilligung s. auch Behling u.a. 2015, S. 15; Pohle/Ghaffari, CR 2017, 489 (490); Wicker 2016, S. $142 f$.

428 S. hierzu Altenhain, in: Matt/Renzikowski 2013, \$ 203 Rn. 35; Kargl, in: Kindhäuser/Neumann/Paeffgen 2017, § 203 Rn. 21a; Cierniak/Niehaus, in: MüKo StGB 2012, § 203 Rn. 56; Wicker 2016, 143.

429 Cierniak/Niehaus, in: MüKo StGB, \203 Rn. 67.

430 Pohle/Ghaffari, CR 2017, 489 (489, 491); Jandt/Roßnagel/Wilke, NSR 2011, 641ff.; Preuß, DuD 2016, $802 f f$.

431 Pohle/Ghaffari, CR 2017, 489 (491). 
verpflichtet wurde; dies gilt nicht für sonstige mitwirkende Personen, die selbst eine in den Absätzen 1 oder 2 genannte Person sind,

2. als im Absatzzgenannte mitwirkende Person sich einer weiteren mitwirkenden Person, die unbefugt ein fremdes, ihr bei der Ausübung oder bei Gelegenheit ihrer Tätigkeit bekannt gewordenes Geheimnis offenbart, bedient und nicht dafür Sorge getragen hat, dass diese zur Geheimhaltung verpflichtet wurde; dies gilt nicht für sonstige mitwirkende Personen, die selbst eine in den Absätzen 1 oder 2 genannte Person sind, oder

3. nach dem Tod der nach Satz 1 oder nach den Absätzen 1 oder 2 verpflichteten Person ein fremdes Geheimnis unbefugt offenbart, das er von dem Verstorbenen erfahren oder aus dessen Nachlass erlangt hat.“

Tatbestandlich fordern $\$ 203$ Abs. 1 und 2 StGB das unbefugte Offenbaren eines fremden Geheimnisses. Täter können die in Abs. 1 und 2 aufgelisteten Personen sein, wenn ihnen das fremde Geheimnis im Rahmen ihrer Tätigkeit nach Abs. 1 und 2 anvertraut oder sonst bekannt geworden ist. Unumstritten war das mündliche Offenbaren. Im Falle verkörperter Geheimnisse war umstritten, ob wie im Falle des mündlichen Offenbarens eine tatsächliche Kenntnisnahme erforderlich ist oder ob die bloße Möglichkeit der Kenntnisnahme ausreicht. ${ }^{432}$ Die Gesetzesbegründung enthält nun die Klarstellung, „dass ein Offenbaren bereits dann gegeben ist, wenn die Möglichkeit der Kenntnisnahme von Geheimnissen besteht“. ${ }^{433}$ Der Streit ist damit zugunsten eines weiten Offenbarungsbegriffs entschieden. Der Einsatz technischer Sicherungsmaßnahmen soll ein Offenbaren ausschließen können. ${ }^{434}$ Damit kann letztlich auch das Unterlassen technisch-organisatorischer Sicherungsmaßnahmen ein Offenbaren begründen.

Nach $\mathbb{2} 203$ Abs. 3 Satz 1 StGB n.F. liegt kein Offenbaren vor, wenn den Abs. 1 und 2 unterfallende Personen Geheimnisse berufsmäßig tätigen Gehilfen oder den ihnen zur Vorbereitung auf den Beruf tätigen Personen zugänglich machen. Im medizinischen Kontext ist dabei insbesondere an medizinische Fachangestellte und medizinisch-technische Assistenten zu denken. Die Regelung hat primär eine klarstellende Funktion. 435 So wurde auch für $\$ 203$ StGB a.F. als ganz herrschende Meinung angenommen, dass bei der Hinzuziehung von Hilfspersonen im beruflichen Kontext keine Offenbarung vorliegt, da die Hilfsperson „unmittelbar an dem konkreten Vertrauensverhältnis teilnimmt“. ${ }^{436}$ Dabei wurde auch darauf abgestellt, dass die Hinzuziehung im

432 Zum Streit s. Altenhain, in: Matt/Renzikowski, \$ 203 Rn. 28f.; Pohle/Ghaffari, CR 2017, 489 (490).

433 BT-Drs. 18/11936, 28. Das „intellektuelle Verstehen“ seitens des Dritten ist nicht erforderlich; Heger, in: Lackner/Kühl 2014, § 203 Rn. 17.

434 So Pohle/Ghaffari, CR 2017, 489 (491); Wicker 2016, S. 133f.; Hartung, VersR 2012, 400 (405); Lenckner/Eisele, in: Schönke/Schröder, § 203 Rn. 19b.

435 BT-Drs. 18/11936, 2.

436 Lenckner/Eisele, in: Schönke/Schröder, \203 Rn. 19a; s. auch Cierniak/Niehaus, in: MüKo StGB, \203 Rn. 53; Wicker 2016, S. 136. 
6 Weitergabe von Informationen an „mitwirkende Personen“

im Rahmen der medizinischen Forschung

Rahmen einer ordnungsgemäßen Berufsausübung erforderlich ist. ${ }^{437}$ Dieses Merkmal findet sich so nicht im Wortlaut von $\mathbb{} 203$ Abs. 3 Satz 1 StGB n.F., dürfte jedoch auch in die neue Vorschrift hineinzulesen sein. Eine Erforderlichkeit soll aber bereits dann gegeben sein, wenn der Berufsgeheimnisträger ein wirtschaftliches Interesse geltend machen kann. ${ }^{438}$

$\mathbb{\$} 203$ Abs. 3 Satz 2 Hs. 1 StGB n.F. enthält eine Fallgruppe, in der zwar ein Offenbaren vorliegt, dieses aber erlaubt ist. Dies ist dann der Fall, wenn fremde Geheimnisse sonstigen Personen offenbart werden, die an der Tätigkeit des Offenbarenden mitwirken. Das Offenbaren muss für die Inanspruchnahme der Tätigkeit der sonstigen mitwirkenden Person erforderlich sein.

Nach $\mathbb{2 0 3}$ Abs. 3 Satz 2 Hs. 2 StGB n.F. ist ein Offenbaren auch dann erlaubt, wenn sonstige mitwirkende Personen sich weiterer Personen bedienen, die an der beruflichen oder dienstlichen Tätigkeit der in Abs. 1 und 2 Genannten mitwirken.

Die mitwirkenden Personen werden durch $\mathbb{} 203$ Abs. 4 Satz 1 StCB n.F. der Strafbarkeit nach $\mathbb{2 0 3}$ StGB unterworfen. ${ }^{439}$ Strafbar macht sich zudem der Berufsgeheimnisträger, wenn er nicht dafür Sorge trägt, dass die sonstige mitwirkende Person zur Geheimhaltung verpflichtet wird, und diese ein Geheimnis unbefugt offenbart. ${ }^{440}$ Gleiches gilt für die mitwirkende Person, wenn sie sich weiterer Personen bedient. ${ }^{441}$ Bestraft wird ferner, wer nach dem Tod einer zur Geheimhaltung verpflichteten ein Geheimnis unbefugt offenbart, das er von der verstorbenen Person oder aus deren Nachlass erfahren hat. ${ }^{442}$

$\mathrm{Zu}$ beachten ist, dass der Berufsgeheimnisträger bereits durch Berufsrecht zur Verschwiegenheit verpflichtet sein kann.

\subsubsection{Verhältnis von $\S 203$ StGB und Datenschutzrecht}

\$ 203 StGB dient zumindest auch dem Schutz des Rechts auf informationelle Selbstbestimmung. ${ }^{443}$

437 Lenckner/Eisele, in: Schönke/Schröder 2014, § 203 Rn. 19a; Jandt/Roßnagel, MedR 2011, 140 (142).

438 BT-Drs. 18/11936, 18.

439 Die Gesetzesbegründung spricht von einer „Verlängerung“ des strafrechtlichen Geheimnisschutzes; BTDrs. 18/11936, 20.

$440 § 203$ Abs. 4 Satz 2 Nr. 1 StGB n.F. Die Pflicht zur Verpflichtung zur Geheimhaltung entfällt, wenn die sonstige mitwirkende Person selbst Berufsgeheimnisträger ist.

$441 \oint 203$ Abs. 4 Satz 2 Nr. 2 StGB n.F.

$442 \oint 203$ Abs. 4 Satz 2 Nr. 3 StGB n.F.

443 Zum Streit s. Altenhain, in: Matt/Renzikowski, \203 Rn. 1; Lenckner/Eisele, in: Schönke/Schröder, \$203 Rn. 3; Tag, in: Dölling/Duttge/König/Rössner, \203 StGB, Rn. 3ff.; Kargl, in: Kindhäuser/Neumann/Paeffgen, \ 203 Rn. 2; Cierniak/Niehaus, in: MüKo StGB, \ 203 Rn. 6; Weidemann, in: BeckOK StGB, \ 203 Rn. 2; Heger, in: Lackner/Kühl, § 203 Rn. 1. 


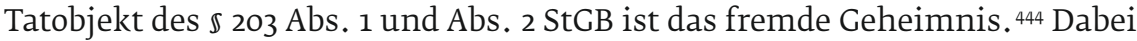
kann es sich um eine beliebige Tatsache handeln. Voraussetzung ist nach einer Auffassung lediglich, dass der Geheimnisträger an der Geheimhaltung der Tatsache ein sachlich begründetes Interesse hat oder haben würde. ${ }^{445}$ Dies wird angenommen für gesundheitliche Verhältnisse, kann aber auch schon anzunehmen sein für die bloße Tatsache, dass sich jemand überhaupt in psychologischer oder ärztlicher Behandlung befindet, was ebenso für die Begleitumstände einer Krankenhausaufnahme gelten kann. ${ }^{446}$ Nach anderer Ansicht liegt ein Geheimnis vor, wenn die Tatsache nur einem beschränkten Personenkreis bekannt ist ${ }^{447}$ und der Betroffene einen Geheimhaltungswillen hat. ${ }^{448}$ Dies wird aber mit dem Erfordernis eines schutzwürdigen ${ }^{449}$ Geheimhaltungsinteresses kombiniert. ${ }^{450}$

Personenbezogene Daten sind nach Art. 4 Nr. 1 DSGVO alle Informationen, die sich auf eine identifizierte oder identifizierbare natürliche Person beziehen. Dies bedeutet, dass nicht jedes personenbezogene Datum ein Geheimnis ist, jedoch handelt es sich bei jedem Geheimnis um ein personenbezogenes Datum. ${ }^{451}$ Wird dieses personenbezogene Datum nach Art. 2 Abs. 1 DSGVO ganz oder teilweise automatisiert verarbeitet oder zwar nichtautomatisiert verarbeitet, aber in einem Dateisystem gespeichert oder wird diese Speicherung angestrebt, so ist ab dem 25. Mai 2018 grundsätzlich die Datenschutz-Grundverordnung anwendbar. Eine Verarbeitung ist dann nur unter den Voraussetzungen von Art. 6 Abs. 1 und eventuell Art. 9 Abs. 2 DSGVO rechtmäßig. Gesundheitsdaten, in Art. 4 Nr. 15 DSGVO definiert als personenbezogene Daten, die sich auf die körperliche oder geistige Gesundheit einer natürlichen Person, einschließlich der Erbringung von Gesundheitsdienstleistungen, beziehen und aus denen Informationen über deren Gesundheitszustand hervorgehen, sind dabei nach Art. 9 DSGVO als besondere Kategorie personenbezogener Daten besonders geschützt.

Eine $\mathbb{1}$ Abs. 3 Satz 2 BDSG a.F. wortgleiche Entsprechung findet sich in $\mathbb{1} 1$ Abs. 2 Satz 3 BDSG-neu. Danach bleibt die Verpflichtung zur Wahrung gesetzlicher Geheimhaltungsvorschriften durch das Bundesdatenschutzgesetz

444 Zusätzlich ist $\S 203$ Abs. 2 Satz 2 StGB zu beachten, der unter bestimmten Umständen Einzelangaben über persönliche und sachliche Verhältnisse dem Geheimnis gleichstellt.

445 Lenckner/Eisele, in: Schönke/Schröder, \$203 Rn. 5, 7.

446 Lenckner/Eisele, in: Schönke/Schröder, \203 Rn. 7.

447 Als „faktisches Begriffselement" Heger, in: Lackner/Kühl, \ 203 Rn. 14.

448 Nach a.A. ist ein Geheimhaltungswille nicht erforderlich; so Weidemann, in: BeckOK StGB, $\$ 203$ Rn. 4a; s. auch Altenhain, in: Matt/Renzikowski, $\$ 203$ Rn. 19f., wonach der Geheimhaltungswille erst auf Ebene der Rechtfertigung relevant ist. S. auch Cierniak/Niehaus, in: MüKo StGB, \ 203 Rn. $12 \mathrm{ff}$.

449 Im Sinn einer „objektiven Geheimhaltungswürdigkeit“ Heger, in: Lackner/Kühl, \$ 203 Rn. 14.

450 S. Cierniak/Niehaus, in: MüKo StGB, \203 Rn. 21; Weidemann, in: BeckOK StGB, \203 Rn. 4a; Kargl, in: Kindhäuser/Neumann/Paeffgen, $\$ 203$ Rn. $6 \mathrm{~b}$ ff.

451 So auch Altenhain, in: Matt/Renzikowski, $\oint 203$ Rn. 17, der einen Personenbezug fordert und diesbezüglich auf $§ 3$ Abs. 1 BDSG a.F. verweist; s. auch Heger, in: Lackner/Kühl, \203 Rn. 14: „Das Geheimnis muss personenbezogen sein." 
6 Weitergabe von Informationen an „mitwirkende Personen“

im Rahmen der medizinischen Forschung

unberührt. Datenschutzrecht und Strafrecht gelten nebeneinander. ${ }^{452}$ Sie haben unterschiedliche Adressaten (Verantwortlicher und Arzt) sowie unterschiedliche Schutzgüter (Patientengeheimnis und informationelle Selbstbestimmung) und Schutzzwecke (Schutz vor Offenbarung und Schutz vor unzulässiger Verarbeitung).453 Daher besteht keine Kollision zwischen $\$ 203$ StGB und datenschutzrechtlichen Vorschriften im Sinne von $\mathbb{S} 1$ Abs. 3 Satz 1 BDSG a.F.

Ist die Verarbeitung nach geltendem Datenschutzrecht rechtmäßig, so folgt daraus deshalb nicht, dass die Offenbarung nicht mehr unbefugt im Sinn von \$ 203 Abs. 1 und 2 StCB ist, auch wenn die Übermittlung an den Empfänger aus datenschutzrechtlicher Sicht rechtmäßig ist. Es ist vielmehr eine gesonderte Offenbarungsbefugnis erforderlich. „Allgemeine datenschutzrechtliche Übermittlungsbefugnisse genügen grundsätzlich nicht". ${ }^{454}$ Anderes gilt aber im Falle von Übermittlungs- und Offenbarungspflichten, die sich auch aus dem Datenschutzrecht ergeben können. Liegt eine solche Pflicht vor, entfällt das Tatbestandsmerkmal des unbefugten Offenbarens. Zudem sollen auch bereichsspezifische Datenschutzvorschriften für den Bereich der medizinischen Forschung anders als allgemeines Datenschutzrecht eine Befugnis zur Über-

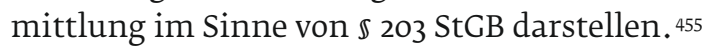

Eine Verschränkung zwischen $₫ 203$ StGB und dem Datenschutzrecht ergibt sich aus der Tatsache, dass auch ein Unterlassen geeigneter technisch-organisatorischer Sicherungsmaßnahmen ein Offenbaren darstellen kann. Hier kann auf die Vorgaben des Art. 32 DSGVO zur Datensicherheit abgestellt werden, um Anhaltspunkte dafür zu finden, wo die Schwelle für ein strafbares Unterlassen liegt. Eine Parallele besteht zudem zwischen dem Erfordernis der Erforderlichkeit in $\$ 203$ Abs. 3 Satz 2 n.F. und dem Grundsatz der Datenminimierung aus Art. 5 Abs. 1 lit. c DSGVO.

Die Befugnisse der Aufsichtsbehörden können nach Art. 9o Abs. 1 DSGVO gegenüber Berufsgeheimnisträgern eingeschränkt werden. Der Gesetzgeber hat diese Möglichkeit durch $\mathbb{2} 29$ Abs. 3 BDSG-neu genutzt. Hier spielt $\mathbb{} 203$ StCB eine konstituierende Rolle. Den dort genannten Personen gegenüber sind die Befugnisse der Aufsichtsbehörde eingeschränkt, „,soweit die Inanspruchnahme der Befugnisse zu einem Verstoß gegen die Geheimhaltungspflichten dieser Personen führen würde“. $\$ 203$ StGB ist damit letztlich auch Grundlage einer signifikanten datenschutzrechtlichen Bevorzugung.

452 Pöttgen 2009, 222f.; Kilian, NJW 1998, 787 (787f.); s. auch Wronka, RDV 2017, 129 (131).

453 Jandt/Roßnagel/Wilke, RDV 2011, 222 (228); Jandt/Roßnagel, MedR 2013, 17ff.; Preuß, DuD 2016, 802 (803).

454 Altenhain, in: Matt/Renzikowski, \ 203 Rn. 38.

455 Pöttgen 2009, 222f.: „Anders stellt sich die Lage jedoch bei den bereichsspezifischen Vorschriften des für den Bereich der medizinischen Forschung relevanten Datenschutzrechts dar, insbesondere bei den Landeskrankenhausgesetzen und den Krebsregistergesetzen. Die in diesen Gesetzen enthaltenen Übermittlungsregeln sind auf die Kollision mit der ärztlichen Schweigepflicht zugeschnitten und können deshalb im Rahmen des $\$ 203$ StGB als Rechtsfertigungsgründe herangezogen werden." 


\section{2 „Berufliche Tätigkeit" des Berufsgeheimnisträgers und „mitwirkende Personen“}

Das fremde Geheimnis muss dem Geheimnisträger in seiner beruflichen Tätigkeit anvertraut oder sonst bekannt geworden sein. Zudem ist fraglich, was unter dem Begriff der „mitwirkenden Personen“ zu verstehen ist, den der $\$ 203$ StGB neu einführt.

\subsection{1 „Berufliche Tätigkeit“}

Das Tatobjekt des fremden Geheimnisses muss in beruflicher Eigenschaft erlangt worden sein - „sei es als Zweck oder Nebenfolge seiner Aufgabenerfüllung“. ${ }^{456}$ Hier differenziert $\$ 203$ StGB zwischen dem anvertrauten und dem sonst bekannt gewordenen fremden Geheimnis. Unter Anvertrauen wird „das Einweihen in ein Geheimnis unter ausdrücklicher Auflage des Geheimhaltens oder unter solchen Umständen, aus denen sich eine Verpflichtung zur Verschwiegenheit ergibt" 457 verstanden. Es ist mithin ein Vertrauensakt erforderlich. ${ }^{45^{8}}$ Erforderlich ist zudem ein innerer Zusammenhang mit der Ausübung seines Berufs. Dies lässt sich aus den Anforderungen an das Wort „als“ in $\$ 203$ Abs. 1 und 2 StGB ableiten. ${ }^{459}$ Ein Vertrag oder eine zivilrechtliche Sonderbeziehung ist nicht erforderlich; das Anvertrauen muss aber ,in der Erwartung erfolgen, dass das Mitgeteilte im Sinne der funktionsgerechten Aufgabenstellung des Empfängers genutzt wird“..460

Auch beim sonst bekannt gewordenen fremden Geheimnis wird ein innerer Zusammenhang zwischen der Aufgabenwahrnehmung des Geheimnisträgers und dem Bekanntwerden verlangt. ${ }^{461}$ Es ist jedoch streitig, ob hier ebenso wie beim anvertrauten Geheimnis ein Vertrauensverhältnis erforderlich ist. ${ }^{462}$

Nicht zur beruflichen Tätigkeit des Berufsgeheimnisträgers gehören andersartige Nebentätigkeiten. Was konkret zur beruflichen Tätigkeit gehört, soll sich „aus dem beruflichen Rollenbild des Täters ergeben“. Berufsfremd sind damit Tätigkeiten, „die überwiegend von anderen Personen professionell wahrgenommen werden. " ${ }^{463}$ Nicht erfasst ist beispielsweise die Tätigkeit eines Arztes, der als Pharmareferent tätig wird. 464

456 Altenhain, in: Matt/Renzikowski, \203 Rn. 23.

457 Weidemann, in: BeckOK StGB, \203 Rn. 12.

458 Cierniak/Niehaus, in: MüKo StGB, \$2 203 Rn. 48.

459 Lenckner/Eisele, in: Schönke/Schröder, \203 Rn. 13; Tag, in: Dölling/Duttge/König/Rössner, \203 StGB, Rn. 40;

Kargl, in: Kindhäuser/Neumann/Paeffgen, \203 Rn. 13f.; Heger, in: Lackner/Kühl, \$203 Rn. 16.

$460 \mathrm{Kargl}$, in: Kindhäuser/Neumann/Paeffgen, \203 Rn. 14.

$461 \mathrm{Kargl}$, in: Kindhäuser/Neumann/Paeffgen, $\$ 203$ Rn. 15.

462 Zum Streit s. Kargl, in: Kindhäuser/Neumann/Paeffgen, \ 203 Rn. 16.

$463 \mathrm{Kargl}$, in: Kindhäuser/Neumann/Paeffgen, \ 203 Rn. 13.

464 So Altenhain, in: Matt/Renzikowski, \203 Rn. 23. 
6 Weitergabe von Informationen an „mitwirkende Personen“

im Rahmen der medizinischen Forschung

\subsection{2 "Mitwirkende Personen“}

Der Begriff der „mitwirkenden Person“ wird mit der Novelle des $\$ 203$ StGB neu in das Strafgesetzbuch eingeführt. Er ist ein Überbegriff und umfasst einerseits den berufsmäßig tätigen Gehilfen und die beim Berufsgeheimnisträger zur Vorbereitung auf den Beruf tätigen Personen und andererseits die sonstigen mitwirkenden Personen. ${ }^{465}$

\subsubsection{Berufsmäßig tätige Gehilfen}

Mit der Novelle des $\mathbb{} 203$ StGB ist der Streit um die Auslegung des Gehilfenbegriffs bezogen auf externe Personen, die nicht in die Organisation des Berufsgeheimnisträgers eingebunden sind, entschieden: Diese unterfallen nicht dem Gehilfenbegriff. So heißt es in der Gesetzesbegründung: Berufsmäßig tätiger Gehilfe ist, „wer innerhalb des beruflichen Wirkungsbereichs des Berufsgeheimnisträgers eine auf dessen berufliche Tätigkeit bezogene unterstützende Tätigkeit ausübt, welche die Kenntnis bzw. die Möglichkeit der Kenntnisnahme fremder Geheimnisse mit sich bringt “. ${ }^{466}$

Die Auslegung des Gehilfenbegriffs bezogen auf interne Personen war aber ebenfalls umstritten. Nach enger Auslegung sollte beispielsweise auch internes EDV-Personal nicht zu den berufsmäßigen Gehilfen zählen, da anders als etwa bei der Krankenschwester hier kein innerer Zusammenhang zur Tätigkeit des Berufsgeheimnisträgers bestünde. ${ }^{467}$ Nach anderer Auffassung ist dies aber der Fall. ${ }^{468}$ Der Streit ist auch nach der Novelle des $\$ 203$ StGB relevant, da er bestimmt, wann die Bevorzugung des $\$ 203$ Abs. 3 Satz 1 StGB n.F. greift und wann auf $\mathbb{} 203$ Abs. 3 Satz 2 StGB n.F. abzustellen ist. Konsens besteht bei der Notwendigkeit eines inneren Zusammenhangs ${ }^{469}$ zwischen der Arbeit des Gehilfen und der „eigentlichen Berufsausübung“ 470 des Geheimnisträgers. Reinigungskräfte, Fahrer und ähnliche Mitarbeiter werden deshalb übereinstimmend nicht als berufsmäßig tätige Gehilfen gewertet. Hier werden lediglich die „äußeren Bedingungen für die fragliche Berufstätigkeit“ geschaffen; ,ihre eigentliche Aufgabe ist inhaltlich nicht hinreichend durch das Arbeitsgebiet

465 S. BT-Drs. 18/11936, 21.

466 BT-Drs. 18/11936, 18, 22.

467 So Altenhain, in: Matt/Renzikowski, $\$ 203$ Rn. 11; Cierniak/Niehaus, in: MüKo StGB, $\ 203$ Rn. 54, wonach etwa die Krankenhausverwaltung außenstehender Dritter sein soll. Angehörige des „technischen Personals“ sollen grundsätzlich ausgeschlossen sein; Cierniak/Niehaus, in: MüKo StGB, § 203 Rn. 124.

468 So zählt etwa Tag, in: Dölling/Duttge/König/Rössner, § 203 StGB, Rn. 19 den EDV-Support durchaus zu den berufsmäßig tätigen Gehilfen. Gleiches gilt bei Kargl, in: Kindhäuser/Neumann/Paeffgen, $\$ 203$ Rn. 38 für „organisatorisch eingebundene EDV-Betreuer"; s. auch Weidemann, in: BeckOK StGB, \$ 203 Rn. 27. S. auch BGH, NJW 1995, 2419 (2420) zum Rechenzentrum eines Krankenhauses.

469 S. aber auch Cierniak/Niehaus, in: MüKo StGB, \ 203 Rn. 123, die von einem „unmittelbaren Zusammenhang“ sprechen. Weidemann, in: BeckOK StGB, \ 203 Rn. 27 fordert neben dem inneren Zusammenhang eine mit der Kenntnisnahme von Geheimnissen verbundene Tätigkeit.

470 Heger, in: Lackner/Kühl, § 203 Rn. 11b. 
der betreffenden Einrichtung geprägt" ${ }^{471} \mathrm{Ob}$ die Tätigkeit ehrenamtlich oder tatsächlich berufsmäßig ausgeübt wird, soll trotz des Wortlauts keine Rolle spielen. ${ }^{472}$ Gefordert wird aber eine organisatorische Anbindung. ${ }^{473}$ Dies stellt auch die Gesetzesbegründung klar, wonach „der Gehilfe nicht selbst seinen Beruf ausüben muss“. ${ }^{474}$

Die Gesetzesbegründung enthält einen Beispielkatalog von „mitwirkenden Tätigkeiten“. Darunter fallen neben Schreibarbeiten und Rechnungswesen auch IT-bezogene Tätigkeiten wie „Einrichtung, Betrieb, Wartung - einschließlich Fernwartung - und Anpassung informationstechnischer Anlagen, Anwendungen und Systeme aller Art“ sowie die „Bereitstellung von informationstechnischen Anlagen und Systemen zur externen Speicherung von Daten“. ${ }^{475}$ Damit dürfte der Streit um die Reichweite des Gehilfenbegriffs bei Personen innerhalb der Sphäre des Berufsgeheimnisträgers in dem Sinn entschieden sein, dass auch interne EDV-Mitarbeiter als Gehilfen zu qualifizieren sind. Dies folgt aus der Tatsache, dass die mitwirkende Person Oberbegriff für den Gehilfen ist. Plausibler ist für die Zukunft also ein (intern) weiter Gehilfenbegriff, dem auch (internes) technisches Personal, Verwaltungsmitarbeiter und Schreibkräfte unterfallen. Ausreichend ist eine Einbindung ,in irgendeiner Weise“" ${ }^{476}$ Reinigungskräfte und Fahrer bleiben aber wohl weiter ausgeschlossen; sie sind nicht „in die Organisation der fraglichen Berufspraxis selbst" 477 eingebunden.

\subsubsection{Sonstige mitwirkende Personen}

Die sonstige mitwirkende Person muss an der beruflichen oder dienstlichen Tätigkeit des Berufsgeheimnisträgers mitwirken. ${ }^{478}$ Die Gesetzesbegründung definiert die sonstigen mitwirkenden Personen als solche, „die zwar an der beruflichen oder dienstlichen Tätigkeit der schweigepflichtigen Person mitwirken, also in diese Tätigkeit in irgendeiner Weise eingebunden werden und Beiträge dazu leisten, allerdings ohne in die Sphäre des Berufsgeheimnisträgers eingegliedert zu sein“. ${ }^{479}$ Der wesentliche Unterschied zum Gehilfen ist also die fehlende Teilhabe an der Sphäre des Berufsgeheimnisträgers. Dafür, wann eine Mitwirkung an der beruflichen oder dienstlichen Tätigkeit des Berufsgeheimnisträgers vorliegt, gibt auch hier der Beispielkatalog in der Geset-

471 Kargl, in: Kindhäuser/Neumann/Paeffgen, §203 Rn. 38; s. auch Cierniak/Niehaus, in: MüKo StGB, § 203 Rn. 124; Weidemann, in: BeckOK StGB, \203 Rn. 27.

472 So Weidemann, in: BeckOK StGB, $\$ 203$ Rn. 27.1; a.A. Cierniak/Niehaus, in: MüKo StGB, $\$ 203$ Rn. 123; Heger, in: Lackner/Kühl, § 203 Rn. 11b.

473 Heger, in: Lackner/Kühl, \$ 203 Rn. 11b.

474 BT-Drs. 18/11936, 21.

475 BT-Drs. 18/11936, 22.

476 BT-Drs. 18/11936, 18, 22.

477 BT-Drs. 18/11936, 18.

478 So der Wortlaut von $\$ 203$ Abs. 3 Satz 2 Hs. 1 StGB.

479 BT-Drs. 18/11936, 22. 
6 Weitergabe von Informationen an „mitwirkende Personen“

im Rahmen der medizinischen Forschung

zesbegründung Anhaltspunkte. Ein externes Schreibbüro kann somit ebenso mitwirkende Person sein wie ein Cloud-Anbieter.

Bezüglich der Grundlage der Mitwirkung heißt es in der Gesetzesbegründung, man wolle „keinen möglichen Rechtsgrund, auf dem eine sonstige Mitwirkung beruhen kann, ausschließen“. ${ }^{480}$ Notwendig sind lediglich die Einbindung in die berufliche Tätigkeit und das Einvernehmen mit der schweigepflichtigen Person.

\subsection{Forschungstätigkeit eines Arztes als „berufliche Tätigkeit“}

Fraglich ist nun einerseits, ob die Forschungstätigkeit des Arztes als berufliche Tätigkeit zu werten ist, und andererseits, ob die Mitwirkung an der Forschungstätigkeit eines Arztes als Mitwirkungshandlung im Sinn des $\$ 203$ StGB n.F. verstanden werden kann.

Nach $\mathbb{1}$ MBO dienen Ärzte der Gesundheit des einzelnen Menschen und der Bevölkerung. ${ }^{481}$ Ihre Aufgabe ist es, „das Leben zu erhalten, die Gesundheit zu schützen und wiederherzustellen, Leiden zu lindern, Sterbenden Beistand zu leisten und an der Erhaltung der natürlichen Lebensgrundlagen im Hinblick auf ihre Bedeutung für die Gesundheit der Menschen mitzuwirken“.

$\$ 15$ Abs. 1 Satz 1 MBO enthält Vorgaben unter anderem für Ärzte, die Forschung mit personenbezogenen Daten durchführen. ${ }^{482}$ Dass die Musterberufsordnung in $\mathbb{1} 15$ überhaupt Regelungen für die ärztliche Forschung enthält, darf bereits als starkes Indiz dafür gelten, dass die Forschungstätigkeit des Arztes als berufliche Tätigkeit zu werten ist.

\subsubsection{Der Arzt im Universitätskrankenhaus}

Das Bundesverfassungsgericht hat festgestellt, dass die Krankenversorgung eine der Universität vom Staat zusätzlich übertragene Aufgabe darstellt: „Ihre Übertragung auf die Universität ist zwar durch die medizinische Forschung und Lehre begründet und bedingt; sie stellt jedoch eine Zusatzaufgabe dar, die in beträchtlichem Maße über den rein wissenschaftlichen Bereich hinausgeht. " 483 Das bedeutet, dass bei Hochschullehrern, die Kranke an Universitätskliniken behandeln, diese Aufgabe neben diejenige tritt, Forschung und Lehre zu betreiben. ${ }^{484}$ Die Verpflichtung zu Forschung und Lehre ergibt sich aus

480 BT-Drs. 18/11936, 22f.; s. auch Pohle/Ghaffari, CR 2017, 489 (492).

481 So auch $₫ 1$ Abs. 1 BÄO.

$482 \bigcirc 15$ Abs. 1 Satz 1 MB0: „Ärztinnen und Ärzte, die sich an einem Forschungsvorhaben beteiligen, bei dem ... Daten verwendet werden, die sich einem bestimmten Menschen zuordnen lassen ....".

483 BVerfG, NJW 1981, 1995 (1996).

484 Lambrecht/Vollmöller, in: Huster/Kaltenborn, $\$ 16$ Rn. 85. 
dem Landeshochschulrecht, z.B. aus $\mathbb{2} 22$ Abs. 2 Satz 2 des Gesetzes für die hessischen Universitätskliniken.

Die wissenschaftliche Forschung gehört damit unzweifelhaft zur beruflichen Tätigkeit des Arztes im Universitätskrankenhaus.

\subsubsection{Der Arzt im sonstigen Krankenhaus}

Der Arzt im sonstigen Krankenhaus ist zur Forschung nicht verpflichtet. Die Nutzung der im Krankenhaus anfallenden Patientendaten für Forschungszwecke wird durch die Landeskrankenhausgesetze aber ausdrücklich gestattet. ${ }^{485}$ Auch die Übermittlung an externe Stellen ist in den Landeskrankenhausgesetzen geregelt und in der Regel an eine Einwilligung des Patienten geknüpft. ${ }^{486}$ Bezogen auf psychisch kranke Personen enthält das Landesrecht entsprechende Regelungen. ${ }^{487}$

Die Forschung des Arztes im sonstigen Krankenhaus ist Teil seiner beruflichen Tätigkeit. Dabei ist es unerheblich, ob die Forschungstätigkeit vom Arbeitgeber angeordnet wurde oder fakultativ erfolgt. Entscheidend ist nur, dass der Arzt „als Arzt“ forscht. Dabei ist zu beachten, dass der Arzt Arzt bleibt, „auch wenn er seinen Beruf nicht als selbstständig praktizierender Arzt oder in einem herkömmlichen Dienstverhältnis, sondern in spezieller Funktion und Rechtsposition ausübt“. ${ }^{488}$ Nicht als Teil der beruflichen Tätigkeit kann lediglich Forschung gelten, die eine andersartige Nebentätigkeit darstellt. Davon dürfte bezogen auf wissenschaftliche Forschung nur auszugehen sein, wenn der Arzt nicht im medizinischen Kontext, also letztlich fachfremd forscht. Ob der Arzt parallel zur Forschungstätigkeit auch im Sinn von $\$ 1$ MBO tätig wird, ist unerheblich. Keine fachfremde Forschung liegt vor, wenn der Arzt als Teil eines Forschungsverbundes seine medizinische Expertise in ein Forschungsvorhaben einbringt, dieses Forschungsvorhaben aber seinen fachlichen Schwerpunkt außerhalb der Medizin hat.

\subsubsection{Der Arzt in der Arztpraxis}

Auch niedergelassene Ärzte sind wie der Arzt im sonstigen Krankenhaus nicht zur Forschung verpflichtet. Dass die Forschungstätigkeit auch des Arztes in der Arztpraxis zu seiner beruflichen Tätigkeit gehört, ergibt sich implizit einerseits aus der Tatsache, dass die Verarbeitung personenbezogener Daten in der Forschung dem Gebot der ärztlichen Schweigepflicht unter-

485 S. beispielhaft $§ 14$ Abs. 1 Saarländisches Krankenhausgesetz; $§ 25$ Abs. 1 Satz 1 Landeskrankenhausgesetz Berlin.

486 S. z.B. Jandt/Roßnagel, MedR 2013, $17 \mathrm{ff}$.

487 S. beispielhaft $\$ 35$ Landesgesetz für psychisch kranke Personen Rheinland-Pfalz.

488 Schelling, in: Spickhoff, $\ 1$ BÄ0, Rn. 7. 
6 Weitergabe von Informationen an „mitwirkende Personen“

im Rahmen der medizinischen Forschung

liegt, ${ }^{489}$ andererseits aus $\mathbb{1} 15$ MBO. Auch hier ist entscheidend, dass der Arzt „als Arzt" forscht. ${ }^{490}$

Voraussetzung ist dabei, dass die Forschungstätigkeit fachlich dem Arztberuf zugeordnet werden kann. Ein Indiz, dass dies nicht so ist, ist etwa, dass die fragliche Tätigkeit klassisch einer anderen Berufsgruppe zugeordnet wird. Aufgrund möglicher Überschneidungen ist jedoch stets eine Betrachtung des konkreten Einzelfalls erforderlich. In jedem Fall ist ein Konnex zum Themenkreis „Medizin“ erforderlich. Sofern die Forschungstätigkeit einen Bezug zu den in $\mathbb{1}$ MBO genannten Aufgaben des Arztes hat, darf dieser als gegeben gelten.

\subsubsection{Forschung als ärztliche Tätigkeit}

Für die Anerkennung von Forschung als ärztlicher Tätigkeit gibt es im Ergebnis keine Unterschiede zwischen der Arbeit eines Arztes in einem Universitätskrankenhaus, in einem sonstigen Krankenhaus und in einer Arztpraxis. In allen Fällen ist die Forschungstätigkeit des Arztes seiner beruflichen Tätigkeit zuzurechnen und deshalb tatbestandlich im Sinn von $\$ 203$ StGB. Entscheidend ist in allen Fällen, dass der forschende Arzt „als Arzt“ tätig wird. Für diese Beurteilung kann als Richtschnur $\mathbb{1} 1 \mathrm{MBO}$ dienen. In allen Fällen erfährt der Arzt ebenso wie bei der Heilbehandlung geheimhaltungsbedürftige Informationen über den Patienten und muss diese Geheimnisse bewahren. Wäre dem nicht so, entstünde zudem eine Schutzlücke bezogen auf den Schutz von Patientendaten, die so nicht gewollt sein kann.

\subsection{Mitwirkung an der Forschungstätigkeit}

Die Mitwirkung an der Forschungstätigkeit eines Arztes ist mithin eine Mitwirkungshandlung im Sinn von $\mathbb{2 0 3}$ StCB. 491

Die Mitwirkung an der Forschungstätigkeit des Arztes ist breit zu verstehen und beinhaltet beispielsweise auch die Auswertung von Datenmassen in externen Rechenzentren. Notwendig ist stets der innere Bezug der Tätigkeit der mitwirkenden Person und der Forschungstätigkeit des Arztes. Zudem muss es für die Tätigkeit der mitwirkenden Person erforderlich sein, dass diese das Geheimnis zur Kenntnis nimmt oder zumindest die Möglichkeit zur Kenntnisnahme erhält.

489 Bekanntgabe der Bundesärztekammer, DÄBL 1989, A-2843 (A-2844).

490 Ansonsten gelten die Ausführungen in Kap. 6.3.2.

491 A.A. wohl Hilgendorf 2004, 93, der einen engen Bezug zur Heiltätigkeit fordert. Nach dieser Ansicht wäre die Forschungstätigkeit des Arztes grundsätzlich keine berufliche Tätigkeit im Sinn von $§ 203$ StGB, da ,sich das Vertrauen des Patienten in erster Linie auf die Heiltätigkeit“ beziehe. 
Liegen diese Voraussetzungen vor, so unterliegt die mitwirkende Person selbst der Strafbarkeit nach $\$ 203$ StCB. Besteht bereits aufgrund eines Gesetzes eine förmliche Verpflichtung einer Person auf die gewissenhafte Erfüllung ihrer Geheimhaltungspflicht bei der Durchführung wissenschaftlicher Forschungsvorhaben, so ist die Person zudem direkt über $\mathbb{2} 203$ Abs. 2 Satz 1 Nr. 6 StGB strafbar. Derartige gesetzliche Verpflichtungen finden sich beispielsweise in $\mathbb{s} 476$ und 487 Abs. 4 StPO, aber auch in $₫ 16$ Abs. 7 BStatG.

\subsection{Ergebnis zur Weitergabe von Informationen an „mitwirkende Personen“}

Strafbar macht sich nach $\mathbb{2} 203$ StCB, wer unbefugt ein fremdes Geheimnis offenbart, das ihm als Berufsgeheimnisträger im Sinne von Abs. 1 und 2 anvertraut worden oder sonst bekannt geworden ist. Hinsichtlich der Strafbarkeit der Offenbarung von Geheimnissen gegenüber externen Fachkräften, die Ärzte in ihrer medizinischen Tätigkeit unterstützten, bestand aufgrund zahlreicher Meinungsverschiedenheiten in der strafrechtlichen Fachliteratur und fehlender obergerichtlicher Rechtsprechung eine große Rechtsunsicherheit. Die Änderung des $\$ 203$ StGB 2017 soll hierzu Rechtsklarheit bringen.

Mit der Novelle des $\$ 203$ StCB wird der Begriff der „mitwirkenden Person“ neu in das Strafgesetzbuch eingeführt. Er ist ein Überbegriff und umfasst einerseits den berufsmäßig tätigen Gehilfen und andererseits die sonstigen mitwirkenden Personen. Wesentliches Unterscheidungsmerkmal zwischen dem Gehilfen und der sonstigen mitwirkenden Person ist dabei die fehlende Einbindung in die Sphäre des Berufsgeheimnisträgers. Eine Offenbarung gegenüber mitwirkenden Personen ist nicht strafbar, wenn die Mitwirkung im Rahmen der Berufstätigkeit des Arztes erfolgt.

Die Forschungstätigkeit des Arztes ist als berufliche Tätigkeit im Sinn von $₫ 203$ StCB zu werten. Entscheidend ist dabei, dass der Arzt „als Arzt “ forscht. Unterschiede zwischen der ärztlichen Tätigkeit in einem Universitätskrankenhaus, einem sonstigen Krankenhaus und einer Arztpraxis bestehen im Ergebnis nicht. In allen Fällen ist die Forschungstätigkeit des Arztes seiner beruflichen Tätigkeit zuzurechnen und deshalb tatbestandlich im Sinn von $\mathbb{2 0 3}$ StCB. Nicht als Teil der beruflichen Tätigkeit kann lediglich Forschung gelten, die eine andersartige Nebentätigkeit darstellt. Davon dürfte nur auszugehen sein, wenn der Arzt nicht im medizinischen Kontext forscht. Damit ist die Mitwirkung an der Forschungstätigkeit eines Arztes eine Mitwirkungshandlung im Sinn von $₫ 203$ Abs. 3 StCB. Eine Offenbarung von Geheimnissen gegenüber der mitwirkenden Person ist nicht strafbar. Die mitwirkende Person unter-

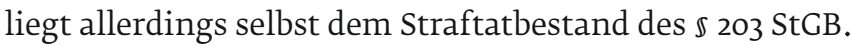




\section{Literatur}

Albrecht, Jan Philipp/Jotzo, Florian: Das neue Datenschutzrecht der EU, Nomos: Baden-Baden 2017.

Arning, Marian A./Forgó, Nikolaus/Krügel, T.: Datenschutzrechtliche Aspekte der Forschung mit genetischen Daten, DuD 2006, 700.

Art. 29-Datenschutzgruppe: Stellungnahme 4/2007 zum Begriff „personenbezogene Daten“, WP 136.

Art. 29-Datenschutzgruppe: Stellungnahme 5/2014 zu Anonymisierungstechniken, WP 216.

Behling, Thorsten B./Borges, Georg u.a.: Schweigepflicht bei der Auslagerung von IT-Dienstleistungen, Kompetenzzentrum Trusted Cloud, Thesenpapier Nr. 7, Februar 2015 (zit.: Behling u.a. 2015).

Bergt, Matthias: Die Bestimmbarkeit als Grundproblem des Datenschutzrechts. Überblick über den Theorienstreit und Lösungsvorschlag, ZD 2015, 365.

Boehme-Neßler, Volker: Das Ende der Anonymität, DuD 2016, 419.

Breyer, Patrick: Personenbezug von IP-Adressen, ZD 2014, 400.

Brink, Stefan/Eckardt, Jens: Wann ist ein Datum ein personenbezogenes Datum?, Anwendungsbereich des Datenschutzrechts, ZD 2015, 205.

Buchner, Benedikt: Grundsätze und Rechtmäßigkeit der Datenverarbeitung unter der DS-GVO, DuD 2016, 155.

Buchner, Benedikt/Kühling, Jürgen: Die Einwilligung in der Datenschutzordnung 2018, DuD 2017, 544.

Calliess, Christian/Ruffert, Matthias (Hrsg.): EUV/AEUV, Kommentar, 5. Aufl., C.H. Beck: München 2016 (zit.: Autor, in: Calliess/Ruffert).

Caspar, Johannes: Geoinformationen und Datenschutz am Beispiel des Internetdienstes Google Street View, DÖV 2009, 965.

Däubler, Wolfgang/Klebe, Thomas/Wedde, Peter/Weichert, Thilo (Hrsg.): Bundesdatenschutzgesetz - Kompakt-Kommentar zum BDSG, 4. Auflage, Bund: Frankfurt a.M. 2014 (zit.: Autor, in: Däubler u.a.).

Danwitz, Thomas von: Die Grundrechte auf Achtung der Privatsphäre und auf Schutz personenbezogener Daten, DuD 2015, 581.

Dölling, Dieter/Duttge, Gunnar/König, Stefan/Rössner, Dieter (Hrsg.): Gesamtes Strafrecht, Kommentar, 4. Aufl., Nomos: Baden-Baden 2017 (zit.: Autor, in: Dölling/Duttge/König/Rössner).

Düsseldorfer Kreis: Beschluss der obersten Aufsichtsbehörden für den Datenschutz im nicht-öffentlichen Bereich am 26./27.11.2009 in Stralsund in Bezug auf IP-Adressen.

Eckhardt, Jens: IP-Adresse als personenbezogenes Datum - neues Öl ins Feuer, CR 2011, 339.

Eckhardt, Jens: Anmerkung zu BGH vom 28.10.2014, CR 2015, 109, CR 2015, 113.

Ehmann, Eugen/Helfrich, Marcus: EG-Datenschutzrichtlinie, Kurzkommentar, Otto Schmidt Verlag: Köln 1999.

Ehmann, Eugen/Selmayr, Martin (Hrsg.): Datenschutz-Grundverordnung, Kommentar, C.H. Beck: München 2017 (zit.: Autor, in: Ehmann/Selmayr).

Ernst, Stefan: Die Einwilligung nach der Datenschutz-Grundverordnung. Anmerkungen zur Definition nach Art. 4 Nr. 11 DS-GVO, ZD 2017, 110.

Geminn, Christian L.: Demokratie zwischen Öffentlichkeit und Privatheit, Verw-Arch 2016, 601-630.

Geminn, Christian L.: Risikoadäquate Regelungen für das Internet der Dienste und Dinge?, DuD 2017, 295.

Geminn, Christian L./Roßnagel, Alexander: „Privatheit“ und „Privatsphäre“ aus der Perspektive des Rechts ein Überblick, IZ 2015, 703.

Gola, Peter (Hrsg.): Datenschutz-Grundverordnung, Kommentar, C.H. Beck: München 2017 (zit.: Autor, in: Gola)

Gola, Peter/Schomerus, Rudolf: Bundesdatenschutzgesetz, Kommentar, 12. Aufl., C.H. Beck: München 2015.

Grabitz, Eberhard/Hilf, Meinhard/Nettesheim, Martin M. (Hrsg.): Das Recht der Europäischen Union, Band I EUV/AEUV, Kommentar, Loseblatt, Beck: München 2017 (zit.: Autor, in: Grabitz/Hilf/Nettesheim)

Haase, Martin S.: Datenschutzrechtliche Fragen des Personenbezugs - Eine Untersuchung des sachlichen Anwendungsbereiches des deutschen Datenschutzrechts und seiner europarechtlichen Bezüge, Mohr Siebeck: Tübingen 2015.

Hänlein, Andreas/Schuler, Rolf: Lehr- und Praxiskommentar Sozialgesetzbuch V, Nomos, 5. Aufl., Baden-Baden 2016 (zit.: Autor, in: Hänlein/Schuler). 
Härting, Nico: Anonymität und Pseudonymität im Datenschutzrecht, NJW 2013, 2065.

Hartung, Jürgen: Datenschutz und Verschwiegenheit bei Auslagerungen durch Versicherungsunternehmen, VersR 2012, 400.

von Heintschel-Heinegg, Bernd (Hrsg.): Beck'scher Online-Kommentar StGB, 37. Edition, C.H. Beck: München 2018 (zit.: Autor, in: BeckOK StGB).

Herbst, Tobias: Rechtliche und ethische Probleme des Umgangs mit Proben und Daten bei großen Biobanken, DuD 2016, 371.

Herbst, Tobias: Was sind personenbezogene Daten?, NVwZ 2016, 902.

Hilgendorf, Eric: Strafrechtliche Probleme beim Outsourcing von Versicherungsdaten, in: Hilgendorf, Eric (Hrsg.), Informationsstrafrecht und Rechtsinformatik, Logos: Berlin 2004.

Hömig, Dieter/Wolff, Heinrich A. (Hrsg.): Grundgesetz für die Bundesrepublik Deutschland, Kommentar, 11. Aufl., Nomos: Baden-Baden 2016 (zit.: Autor, in: Hömig/Wolff).

Hoeren, Thomas/Sieber, Ulrich/Holznagel, Bernd (Hrsg.): Handbuch Multimedia-Recht - Rechtsfragen des elektronischen Geschäftsverkehrs, Loseblatt, C.H. Beck: München (zit. Autor, in: Hoeren/Sieber/Holznagel).

Hofmann, Johanna M./Johannes, Paul C.: DS-GVO: Anleitung zur autonomen Auslegung des Personenbezugs, ZD 2017, 221.

Hornung, Gerrit: Der Personenbezug biometrischer Daten, DuD 2004, 429.

Hornung, Gerrit/Hofmann, Kai: Die Auswirkungen der europäischen Datenschutzreform auf die Markt- und Meinungsforschung, ZD-Beilage 4/2017, 1.

Huster, Stefan/Kaltenborn, Markus (Hrsg.): Krankenhausrecht, 2. Aufl., C.H. Beck: München 2017 (zit.: Autor, in: Huster/Kaltenborn).

Jandt, Silke/Roßnagel, Alexander: Qualitätssicherung im Krankenhaus, MedR 2011, 140.

Jandt, Silke/Roßnagel, Alexander: Factoring von Forderungen aus Behandlungsverträgen der Krankenhäuser datenschutzrechtlich zulässig?, MedR 2013, 17-23.

Jandt, Silke/Roßnagel, Alexander/Wilke, Daniel: Krankenhausinformationssysteme im Gesundheitskonzern, RDV 2011, 222.

Jandt, Silke/Roßnagel, Alexander/Wilke, Daniel: Outsourcing im Medizinbereich, NSR 2011, 641.

Jarass, Hans D.: Charta der Grundrechte der Union, 3. Aufl., C.H. Beck: München 2016.

Jauernig, Othmar (Begr.): Bürgerliches Gesetzbuch, Kommentar, 16. Aufl., C.H. Beck: München 2015 (zit.: Autor, in: Jauernig).

Joecks, Wolfgang/Miebach, Klaus (Hrsg.): Münchener Kommentar zum StGB, Bd. 4, 3. Aufl., C.H. Beck: München 2017 (zit.: Autor, in: MüKo StGB).

Johannes, Paul C./Richter, Philipp: Privilegierte Verarbeitung im BDSG-E, DuD 2017, 300.

Karg, Moritz: Die Rechtsfigur des personenbezogenen Datums - ein Anachronismus des Datenschutzes?, ZD 2012, 255.

Karg, Moritz: Anonymität, Pseudonymisierung und Personenbezug revisited?, DuD 2015, 520.

Kartheuser, Ingemar/Gilsdorf, Friedrich: EuGH: Dynamische IP-Adressen können personenbezogene Daten sein, MMR-Aktuell 2016, 382533

Kilian, Wolfgang: Medizinische Forschung und Datenschutzrecht, NJW 1998, 787-791.

Kindhäuser, Urs/Neumann, Ulfried/Paeffgen, Hans-Ullrich (Hrsg.): Strafgesetzbuch, Kommentar, 5. Aufl., Nomos: Baden-Baden 2017 (zit.: Autor, in: Kindhäuser/Neumann/Paeffgen).

Klar, Manuel: Datenschutzrecht und die Visualisierungen des öffentlichen Raums, Lit: Münster 2012.

Knopp, Michael: Pseudonym - Grauzone zwischen Anonymisierung und Personenbezug, DuD 2015, 527.

Kroschwald, Steffen: Informationelle Selbstbestimmung in der Cloud, Springer: Berlin u.a. 2015.

Krügel, Tina: Das personenbezogene Datum nach der DS-GVO - Mehr Klarheit und Rechtssicherheit, ZD 2017, 455 .

Krüger, Stefan/Maucher, Svenja-Ariane: Ist die IP-Adresse wirklich ein personenbezogenes Datum? - Ein falscher Trend mit großen Auswirkungen auf die Praxis, MMR 2011, 433.

Kühling, Jürgen/Buchner, Benedikt: Datenschutz-Grundverordnung, Kommentar, 2. Aufl. C.H. Beck: München 2018 (zit.: Autor, in: Kühling/Buchner). 
Kühling, Jürgen/Klar, Manuel: Unsicherheitsfaktor Datenschutzrecht - Das Beispiel des Personenbezugs und der Anonymität, NJW 2013, 3611.

Kühling, Jürgen/Klar, Manuel: Anmerkung zu EuGH, Urt. v. 19.10.2016, C-582/14, ZD 2017, 24, ZD 2017, 27.

Kühling, Jürgen/Martini, Mario u.a.: Die Datenschutz-Grundverordnung und das nationale Recht, Verlagshaus Monsenstein und Vannerdat: Münster 2016.

Lackner, Karl/Kühl, Kristian (Hrsg.): Strafgesetzbuch, Kommentar, 28. Aufl., C.H. Beck: München 2014 (zit.: Autor, in: Lackner/Kühl).

Laue, Philip/Nink, Judith/Kremer, Sacha: Das neue Datenschutzrecht in der betrieblichen Praxis, Nomos: Baden-Baden 2016.

Marnau, Ninja: Anonymisierung, Pseudonymisierung und Transparenz für Big Data, DuD 2016, 428.

Masing, Johannes: Herausforderungen des Datenschutzes, NJW 2012, 2305.

Matt, Holger/Renzikowski, Joachim (Hrsg.): Strafgesetzbuch, Kommentar, Vahlen: München 2013 (zit.: Autor, in: Matt/Renzikowski).

Maunz, Theodor/Dürig, Günter (Hrsg.): Grundgesetz, Loseblatt, C.H. Beck: München 2016 (zit.: Autor, in: Maunz/Dürig).

Meyer, Jürgen (Hrsg.): Charta der Grundrechte der Europäischen Union, Kommentar, 4. Aufl., Nomos: Baden-Baden 2014 (zit.: Autor, in: Meyer).

Meyerdierks, Per: Sind IP-Adressen personenbezogene Daten?, MMR 2009, 8.

Moos, Flemming/Rothkegel, Tobias: Anmerkung zu EuGH, Urt. v. 19.10.2016, C-582/14, MMR 2016, 842, MMR 2016, 845 .

Nink, Judith/Pohle, Jan: Die Bestimmbarkeit des Personenbezugs. Von der IP-Adresse zum Anwendungsbereich der Datenschutzgesetze, MMR 2015, 563.

Paal, Boris P./Pauly, Daniel (Hrsg.): Datenschutz-Grundverordnung, Kommentar, 2. Aufl., C.H. Beck: München 2018 (zit.: Autor, in: Paal/Pauly).

Pahlen-Brandt, Ingrid: Datenschutz braucht scharfe Instrumente - Beitrag zur Diskussion um „personenbezogene Daten", DuD 2008, 34.

Pahlen-Brandt, Ingrid: Zur Personenbezogenheit von IP-Adressen, K \& R 2008, 286.

Plath, Kai-Uwe (Hrsg.): BDSG/DSGVO, Kommentar, 2. Aufl., Dr. Otto Schmidt: Köln 2016 (zit.: Autor, in: Plath).

Pohle, Jan/Ghaffari, Sheila: Die Neufassung des $\$ 203$ StGB - der Befreiungsschlag für IT-Outsourcing am Beispiel der Versicherungswirtschaft?!, CR 2017, 489.

Pöttgen, Nicole: Medizinische Forschung und Datenschutz, Peter Lang: Frankfurt am Main 2009.

Preuß, Tamina: Die Strafbarkeit von Berufsgeheimnisträgern nach $\$ 203$ StGB beim Cloud Computing, DuD 2016, 802.

Roßnagel, Alexander (Hrsg.): Handbuch Datenschutzrecht - Die neuen Grundlagen für Wirtschaft und Verwaltung C.H. Beck: München 2003 (zit.: Autor, in: Roßnagel 2003).

Roßnagel, Alexander: Datenschutz in einem informatisierten Alltag - Gutachten, Friedrich Ebert Stiftung: Berlin 2007.

Roßnagel, Alexander: Modernisierung des Datenschutzes - Nicht die Definition von Personendaten muss geändert werden, sondern die Anforderungen an ihren Schutz, digma 2011, 160.

Roßnagel, Alexander (Hrsg.): Beck'scher Kommentar zum Recht der Telemediendienste - Telemediengesetz, Jugendmedienschutz-Staatsvertrag (Auszug), Signaturgesetz, Signaturverordnung, Vorschriften zum elektronischen Rechts- und Geschäftsverkehr, C.H. Beck: München 2013 (zit.: Autor, in: Roßnagel 2013).

Roßnagel, Alexander (Hrsg.): Europäische Datenschutz-Grundverordnung, Vorrang des Unionsrechts - Anwendbarkeit des nationalen Rechts, Nomos: Baden-Baden 2017 (zit.: Autor, in: Roßnagel 2017).

Roßnagel, Alexander: Datenschutzaufsicht nach der EU-Datenschutz-Grundverordnung, Springer Verlag, Wiesbaden 2017.

Roßnagel, Alexander: Gesetzgebung im Rahmen der Datenschutz-Grundverordnung - Aufgaben und Spielräume des deutschen Gesetzgebers?, DuD 2017, 277.

Roßnagel, Alexander (Hrsg.): Das neue Datenschutzrecht - Europäische Datenschutz-Grundverordnung und deutsche Datenschutzgesetze, Nomos: Baden-Baden 2018 (zit.: Autor, in: Roßnagel 2018). 
Roßnagel, Alexander/Banzhaf, Jürgen/Grimm, Rüdiger: Datenschutz im Electronic Commerce, Recht und Wirtschaft: Heidelberg 2003.

Roßnagel, Alexander/Geminn, Christian L./Jandt, Silke/Richter, Philipp: Datenschutzrecht 2016 - „Smart“ genug für die Zukunft? Ubiquitous Computing und Big Data als Herausforderungen des Datenschutzrechts, kassel university press, Kassel 2016.

Roßnagel, Alexander/Kroschwald, Steffen: Was wird aus der Datenschutzgrundverordnung? Die Entschließung des Europäischen Parlaments über ein Verhandlungsdokument, ZD 2014, 495.

Roßnagel, Alexander/Nebel, Maxi: (Verlorene) Selbstbestimmung im Datenmeer - Privatheit im Zeitalter von Big Data, DuD 2015, 455.

Roßnagel, Alexander/Pfitzmann, Andreas/Garstka, Hansjürgen: Modernisierung des Datenschutzrechts - Gutachten im Auftrag des Bundesministeriums des Innern, Berlin 2002.

Roßnagel, Alexander/Scholz, Philip: Datenschutz durch Anonymität und Pseudonymität, Rechtsfolgen der Verwendung anonymer und pseudonymer Daten, MMR 2000, 721.

Sachs, Ulrich: Datenschutzrechtliche Bestimmbarkeit von IP-Adressen, CR 2010, 547.

Säcker, Franz I./Rixecker, Roland/Oetker, Hartmut/Limperg, Bettina (Hrsg.): Münchener Kommentar zum Bürgerlichen Gesetzbuch, Bd. 4, 7. Aufl., C.H. Beck: München 2016 (zit.: Autor, in: MüKo BGB).

Schaar, Katrin: DS-GVO: Geänderte Vorgaben für die Wissenschaft. Was sind die neuen Rahmenbedingungen und welche Fragen bleiben offen?, ZD 2016, 224.

Schaar, Peter: Datenschutz im Internet - Die Grundlagen, C.H. Beck: München 2002.

Schaffland, Hans-Jürgen/Wiltfang, Noeme: Bundesdatenschutzgesetz - BDSG - Ergänzbarer Kommentar nebst einschlägigen Rechtsvorschriften, Loseblatt, Berlin.

Schantz, Peter: Datenschutz-Grundverordnung, NJW 2016, 1841.

Schantz, Peter/Wolff, Heinrich A.: Das neue Datenschutzrecht - Datenschutz-Grundverordnung und Bundesdatenschutzgesetz in der Praxis, C.H. Beck: München 2017 (zit.: Autor, in: Schantz/Wolff).

Schneider, Uwe K.: Sekundärnutzung klinischer Daten - Rechtliche Rahmenbedingungen, Schriftenreihe der TMF Bd. 12, Medizinisch Wissenschaftliche Verlagsgesellschaft: Berlin 2015.

Schönke, Adolf/Schröder, Horst (Hrsg.): Strafgesetzbuch, Kommentar, 29. Aufl., C.H. Beck: München 2014 (zit.: Autor, in: Schönke/Schröder).

Schulze, Reiner (Hrsg.): Bürgerliches Gesetzbuch, Kommentar, 9. Aufl., Nomos: Baden-Baden 2017 (zit.: Autor, in: Schulze).

Simitis, Spiros (Hrsg.): Bundesdatenschutzgesetz, Kommentar, 8. Aufl., Nomos: Baden-Baden 2014 (zit.: Autor, in: Simitis).

Simitis, Spiros/Hornung, Gerrit/Spiecker gen. Döhmann, Indra (Hrsg.), Datenschutzrecht - DSGVO mit BDSG, Nomos, Baden-Baden 2018.

Spickhoff, Andreas (Hrsg.): Medizinrecht, 2. Aufl., C.H. Beck: München 2014 (zit.: Autor, in: Spickhoff).

Stiemerling, Oliver/Hartung, Jürgen: Datenschutz und Verschlüsselung - Wie belastbar ist Verschlüsselung gegenüber dem Anwendungsbereich des Datenschutzrechts?, CR 2012, 60.

Sydow, Gernot (Hrsg.): Europäische Datenschutzgrundverordnung, Kommentar, Nomos: Baden-Baden 2017 (zit.: Autor, in: Sydow).

Taeger, Jürgen/Gabel, D. (Hrsg.): Kommentar zum BDSG und zu den Datenschutzvorschriften des TKG und TMG, 2. Aufl., Frankfurt a.M. 2013 (zit.: Autor, in: Taeger/Gabel)

Voigt, Paul: Datenschutz bei Google, MMR 2009, 377.

Weichert, Thilo: Cloud Computing und Datenschutz, DuD 2010, 679.

Wicker, Magda: Cloud Computing und staatlicher Strafanspruch, Nomos: Baden-Baden 2016.

Wolff, Heinrich A./Brink, Stefan (Hrsg.): Datenschutzrecht, Kommentar, 23. Edition, C.H. Beck: München 2018 (zit.: Autor, in: Wolff/Brink).

Wronka, Georg: Datenschutzrechtliche Aspekte des „neuen“ \& 203 StGB, RDV 2017, 129.

Ziebarth, Wolfgang: Automatisierte Erfassung und Verarbeitung von Kfz-Kennzeichen zu Fahndungszwecken, CR 2015; 687. 